\title{
Entre la judicialización y la atribución de responsabilidades. Encuadres en la cobertura noticiosa de escándalos políticos en Chile (2014-2016)
}

\section{Between the judicialization and the attribution of responsibilities. Frames in the news coverage of political scandals in Chile (2014-2016)}

\author{
René-Eduardo Jara-Reyes; Matías Berrios; Deborah Moreno
}

Cómo citar este artículo:

Jara-Reyes, René-Eduardo; Berrios, Matías; Moreno, Deborah (2020). “Entre la judicialización y la atribución de responsabilidades. Encuadres en la cobertura noticiosa de escándalos políticos en Chile (2014-2016)". El profesional de la información, v. 29, n. 2, e290224.

https://doi.org/10.3145/epi.2020.mar.24

Artículo recibido el 25-02-2019

Aceptación definitiva: 16-12-2019

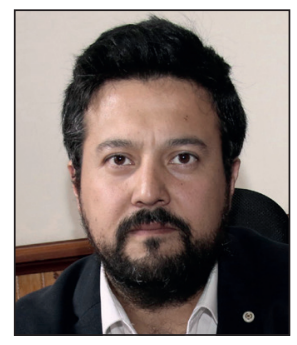

René-Eduardo Jara-Reyes https://orcid.org/0000-0001-8203-5917

Universidad de Santiago de Chile

Escuela de Periodismo

Facultad de Humanidades

Av. Ecuador, 3650.

Santiago (Región Metropolitana), Chile

rene.jara@usach.cl

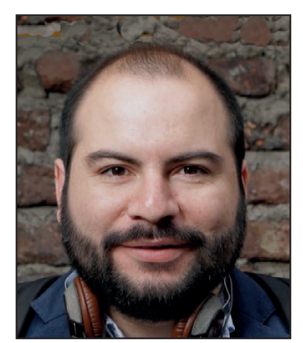

Matías Berrios

https://orcid.org/0000-0001-7063-391X

Universidad de Santiago de Chile

Escuela de Periodismo

Facultad de Humanidades

Av. Ecuador, 3650.

Santiago (Región Metropolitana), Chile

matias.berrios@usach.cl

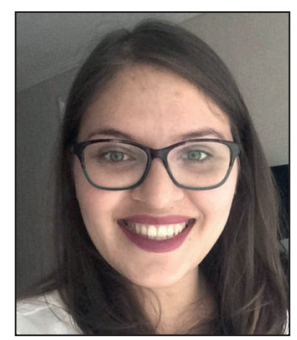
Deborah Moreno
https://orcid.org/0000-0001-7259-2349
Universidad de Santiago de Chile
Escuela de Periodismo
Facultad de Humanidades
Av. Ecuador, 3650.
Santiago (Región Metropolitana), Chile
deborah.moreno@usach.cl

\section{Resumen}

La mediatización de los escándalos políticos se ha vuelto un fenómeno corriente en los más diversos sistemas políticos. No obstante, sabemos poco aún cómo los medios encuadran estas informaciones. Para responder esta interrogante, hemos desarrollado un estudio descriptivo sobre una serie de escándalos políticos recientes denunciados en la prensa chilena. Tomando como marco temporal las noticias publicadas entre 2014 y 2016, hemos analizado el total de informaciones ( $n=10011$ ) publicadas por una muestra de once medios nacionales, sobre siete escándalos políticos que se produjeron durante el periodo. Nos hemos servido del programa QDAMiner-Wordstat para detectar frame terms, a partir de los cuales hemos podido identificar dos grandes encuadres: uno referido a la "judicialización de los casos" y otro referido a la "atribución de responsabilidades". Se presentan y discuten los resultados, examinando algunas implicaciones del ejercicio para la comprensión de los escándalos políticos en sí mismos y la forma en que la prensa periódica chilena los presenta y difunde. 


\title{
Palabras clave
}

Encuadres; Encuadres noticiosos; Periodismo; Prensa nacional; Prensa regional; Diarios; Escándalos políticos; Judicialización; Atribución de responsabilidades; Chile.

\begin{abstract}
The mediatization of political scandals has become a common phenomenon in the most diverse political systems. However, we know little about how the media frame this information. To answer this question, we have developed a descriptive study on a series of recent political scandals denounced in the Chilean press. Taking as a temporary framework the news published between 2014 and 2016, we analyzed the total information $(n=10011)$ published by a sample of eleven national media, on seven political scandals that occurred during the period. We have used the QDAMiner-Wordstat program to detect frame terms, from which we have been able to identify two large frames: one referring to the "Judicialization of cases" and the other referred to the "Attribution of responsibilities". The results are presented and discussed, examining some implications of the exercise for the understanding of the political scandals themselves and the way in which the Chilean periodic press presents and disseminates them.
\end{abstract}

\section{Keywords}

Frames; News frames; Journalism; Regional press; National press; Newspapers; Political scandals; Judicialization; Attribution of responsibilities; Chile.

\section{Introducción}

Desde hace algunas décadas la cobertura de noticias sobre escándalos políticos se ha incrementado significativamente (Allern; Von-Sikorski, 2018, p. 3014). Junto con este incremento se ha llevado a cabo una discusión teórica y conceptual sobre el fenómeno. No obstante, aún hay pocos trabajos que analicen la cuestión de la cobertura de prensa de estos casos, sobre todo en el contexto latinoamericano. Para examinar este problema el caso chileno resulta de particular interés, ya que el país gozaba de indicadores altos de confianza en las instituciones y bajos niveles de percepción de corrupción. Ambas tendencias se han revertido dramáticamente durante los últimos años. Las características del mercado de la prensa periódica, su homogeneidad, centralismo y segmentación son factores relevantes a considerar en el estudio de la cobertura de este tipo de informaciones.

\subsection{Mediatización de los escándalos políticos. Estado de la cuestión}

Ya sea en la lógica más funcionalista (Markovits; Silverstein, 1988) o simbólico-política (Thompson, 2001), la comprensión del escándalo político tiene como eje central el análisis de su mediatización. En este plano la evaluación de políticos y las consecuencias electorales aparecen como los temas más frecuentemente estudiados (Von-Sikorski, 2018). Allern y Von-Sikorski (2018, p. 3020) han identificado al menos cuatro tipos de efectos relevantes de la mediatización de los escándalos:

- pueden afectar negativamente a la actitud de los ciudadanos hacia los políticos envueltos en escándalos;

- pueden afectar a ciertas formas de participación política como en las elecciones e influenciar los resultados electorales;

- pueden afectar a la confianza política de los ciudadanos y, de manera general, su satisfacción con la democracia;

- las noticias que informan sobre escándalos políticos pueden influenciar las percepciones públicas de los mismos medios informativos.

Los escándalos políticos mediatizados son un hecho regular durante las campañas electorales, influenciando los resultados de manera diversa (Fernández-Vázquez; Barberá; Rivero, 2016). Si bien los escándalos parecen afectar en general de manera negativa a los acusados, este efecto se ve matizado por las características del candidato y sus conductas, las actitudes previas, el contexto y el tipo de escándalo (Von-Sikorski; Knoll, 2019). Las organizaciones informativas pueden incluso incrementar su legitimidad denunciando estos escándalos como violaciones o transgresiones (Allern; Pollack, 2012), validando con ello las normas sociales (Jacobsson; Löfmark, 2008, p. 212). Los escándalos pueden ser vistos entonces como una posibilidad para que los medios se validen y logren una mayor legitimidad frente a sus audiencias.

Dentro de este campo de estudio, la cobertura informativa es uno de los puntos que no ha sido suficientemente analizado. Stein-de-Sparvieri (2013) señala que los pocos estudios existentes se han centrado en conocer las tendencias, intensidad o frecuencia de cobertura, así como el rol partidista que asume el medio de comunicación a la hora de juzgar, defender o atacar posturas (Puglisi; Snyder, 2011; Palau; Davesa, 2013; Romano, 2016). En este escenario se ha descubierto que los periódicos suelen responder a los gustos partidarios de los lectores sólo por los escándalos locales. Por ejemplo, la prensa estadounidense se hace eco de problemas que suceden fundamentalmente en los estados (es decir, escándalos locales), y no de lo ocurrido a nivel nacional (Puglisi; Snyder, 2011).

Los escándalos políticos pueden ser una posibilidad para que los medios se validen y logren una mayor legitimidad frente a sus audiencias 


\subsection{Escándalos políticos en Iberoamérica y en Chile}

Si bien existen estudios sobre escándalos políticos en Europa, Norteamérica y Asia, los trabajos en el contexto iberoamericano son aún escasos.

Para el caso argentino, Romano (2016) realizó un estudio cuantitativo-cualitativo de recursos lingüísticos y técnicas discursivas, para detectar los propósitos que existían a raíz de un caso particular ocurrido en el gobierno de Néstor Kirchner (2003-2007). En la misma línea de trabajo, Palau y Davesa (2013) realizaron un análisis de la cobertura mediática de los escándalos de corrupción en España entre 1996 y 2009. Los autores plantearon que los periódicos más leídos en esa nación -El país y El mundo- realizan una cobertura de escándalos siguiendo orientaciones políticas distintas. Es decir,

"se identifican diferencias importantes entre ambos periódicos en la cobertura que realizan de los casos de corrupción en función del partido político implicado (PP O PSOE)" (Palau; Davesa, 2013, p. 1).

En el caso chileno los escándalos políticos ligados a temas de corrupción han aparecido con mucha fuerza durante los últimos años, pese a que se conocieron en el pasado casos emblemáticos (Sunkel, 2004), que marcaron un antes y un después en la cobertura de este tipo de noticias políticas. El último ciclo de denuncias se produjo entre 2014 y 2016 , cuando se multiplicaron los casos denunciados. La caída en los índices de confianza hacia los políticos (Gamboa; Segovia, 2016) y la irrupción de la corrupción como un tema central de interés para la opinión pública (CEP, 2014; 2015; 2016) dan cuenta de la relevancia que han cobrado estos temas en Chile.

\subsection{Homogeneidad, orientación comercial y centralización. La prensa en Chile}

La cobertura informativa de los escándalos puede ser explicada en parte a partir de las propias características del sistema de prensa chileno. La concentración de la propiedad de los medios en grandes empresas o consorcios periodísticos de Chile, ligados a los grupos mediáticos EI Mercurio SAP y Copesa, ha afectado gravemente el pluralismo del sistema mediático. Un grupo importante de autores señala que la concentración ha tendido a homogenizar los contenidos, ideas y opiniones aportadas al debate público, sosteniendo que Chile sería un caso emblemático de concentración y homogeneidad (Sunkel; Geoffroy, 2001; Mönckeberg, 2009; Mellado-Ruiz; Humanes, 2017), donde la competencia es un atributo escaso (Valenzuela; Arriagada, 2009). Para otros autores, la homogeneidad se observa sobre todo en las rutinas y pautas de trabajo, sin que ello constituya

"una pérdida de la diversidad de los puntos de vista expresados por sus fuentes, la que varía de caso en caso" (Gronemeyer; Porath, 2015).

Finalmente, estudios recientes han levantado además evidencia sobre ciertos sesgos políticos de los principales periódicos, en el tratamiento que dan a los presidentes de la república de diversas tendencias políticas (Navia; Osorio, 2015), los cuales en cualquier en caso no contradicen la tendencia general a la homogeneización de los contenidos.

La segunda característica del sistema es su marcada orientación comercial. Algunos autores lo identifican como un modelo comercial segmentado (Stange-Marcus et al., 2018). Los mismos grupos mediáticos crean productos centrados en públicos heterogéneos a partir de roles periodísticos particulares, sobre todo en el campo de las noticias políticas (Mellado-Ruiz; López-Rabadán; Elortegui, 2017; Hallin; Mellado, 2018).

En tercer lugar se observa que la agenda de los medios está profundamente centralizada en los sucesos que ocurren en la capital. Algunos autores señalan que se debe

“a la concentración de poder económico y político en pocas organizaciones" (Arriagada et al., 2015)

es necesario agregar la centralización que existe en términos geográficos -principalmente en la Región Metropolitanade las empresas informativas (Sunkel; Geoffroy, 2001), lo que va también en detrimento de la prensa de regiones (Salinas-Muñoz et al., 2015). En síntesis, la homogeneidad, la orientación comercial y la concentración geográfica de la prensa pueden repercutir en la cobertura noticiosa de los escándalos políticos, sobre todo si consideramos que varios de estos estos casos tienen como escenario las regiones del país.

La homogeneidad, la orientación comercial y la concentración geográfica de la prensa pueden repercutir en la cobertura noticiosa

\subsection{Preguntas de investigación}

Tomando en cuenta los antecedentes revisados, el marco teórico señalado y el contexto del mercado chileno de la prensa periódica, este artículo plantea las siguientes preguntas:

P1. ¿Cómo encuadra la prensa chilena los escándalos políticos que han sucedido entre 2014 y 2016 ?

P2. ¿Qué encuadres son los más utilizados y para qué tipo de escándalos se emplean?

P3. ¿Qué encuadres privilegian los diferentes medios de prensa según su orientación y su vecindad con estos casos? 


\section{Organización metodológica}

\subsection{Tipo de investigación y técnica de análisis}

Para responder a estas preguntas, el presente artículo realiza un estudio descriptivo de los encuadres noticiosos predominantes en un corpus de noticias construido para esos fines. Dada la cantidad y la extensión de este corpus, se ha decidido utilizar técnicas de estudio de encuadres asistido por programas informáticos. Estos estudios tienen como base la técnica llamada frame mapping. Esta técnica posibilita

"la determinación de un conjunto adecuado de palabras clave utilizadas por las partes interesadas para articular sus marcos" (Miller, 1997).

La ventaja del método consiste en reducir las variables subjetivas en la búsqueda de los encuadres presentes típicamente en los enfoques puramente cualitativos utilizados en los estudios de encuadres o news frames. A partir de este trabajo de explotación de texto, se busca extraer las dimensiones subyacentes a los conceptos, analizando cómo se agrupan los conceptos entre sí, mediante el análisis estadístico de co-ocurrencia del concepto. Con el mapa semántico detectado (o agrupación de palabras mediante la co-ocurrencia de conceptos clave), se pueden inferir encuadres. Posteriormente se deberá dar nombre a estas agrupaciones de co-ocurrencias,

"llegando así a la identificación de los diferentes encuadres noticiosos" (Matthes; Kohring, 2008).

Se trata de un diseño de investigación inductivo en dos fases:

- en la primera se realizan mediciones estadísticas, propias de un corpus lingüístico;

- en la segunda se realiza un análisis cualitativo de los resultados.

Para el análisis de contenido utilizamos el paquete informático de la empresa Provalis Research, QDA Miner-Wordstat en su versión de pago. El programa procesa el conjunto de palabras mediante un análisis estadístico de correspondencias. Este análisis permite construir una matriz tridimensional con los valores de los eigen vectores de las palabras (Fenoll; Rodríguez-Ballesteros, 2017). Concluido este proceso, Wordstat arroja un número finito de grupos de palabras. "La matriz tridimensional" a la que hacen referencia Fenoll y Rodríguez-Ballesteros (2017) son estos grupos de palabras, los que finalmente se convertirán en los encuadres detectados por el software y los que utilizan los medios de comunicación.

\subsection{Frames genéricos y particulares para escándalos políticos}

La bibliografía sobre encuadres de noticias (news frames) realiza una distinción entre dos modelos establecidos por De-Vreese (2005) para clasificarlos: encuadres genéricos y específicos.

Los encuadres genéricos son aquellos aplicables a diferentes temas, incluso a lo largo del tiempo y potencialmente en diferentes contextos culturales. Semetko y Valkenburg (2002) identifican cinco tipos:

- conflicto entre individuos, grupos o instituciones;

- interés humano, con el que se dramatiza o se le da una perspectiva emocional a la información;

- consecuencias económicas;

- atribución de responsabilidad, poniendo el énfasis en que una institución o un individuo es responsable de causar o resolver un asunto;

- moralidad, centrado en una lectura moral o religiosa del problema.

También es posible identificar estudios que han construido encuadres específicos para estudiar diferentes temas, como por ejemplo la inmigración (Igartua; Muñiz; Cheng, 2005). En el caso particular de los escándalos de corrupción política, Zamora y Marín-Albadalejo (2010) identifican seis tipos de encuadres:

- personificación;

- comparación;

- corrupción generalizada;

- reacción popular;

- botín conquistado;

- depuración de responsabilidades.

Si tomamos en consideración los encuadres genéricos propuestos por Semetko y Valkenburg y por Zamora y Marín-Albadalejo para estudiar los escándalos políticos, podemos observar que existe alguna cercanía entre los encuadres propuestos:

- el encuadre genérico "atribución de responsabilidades" se corresponde bastante al específico "depuración de responsabilidades";

- el genérico "de interés humano" se corresponde con el encuadre "personificación". La cuestión del conflicto corresponde en este caso a la idea de reacción popular;

- el carácter agonístico o de conflicto, de estrategia o de juego político que está presente en el primer encuadre genérico conflicto, se recoge también en el encuadre "comparación" y "botín conquistado". Este último rescata la dimensión de arma política, que constituye uno de los elementos que según la bibliografía es relevante para investigar estos casos. 


\subsection{Diseño muestral y variables de análisis}

Para realizar nuestro estudio hemos constituido un corpus de análisis en base a la cobertura de siete escándalos políticos en una muestra representativa de los medios de prensa escrita. Para su selección, hemos privilegiado dos tipos de escándalos:

- casos de cobertura intensa e interés general: (casos Caval, Corpesca, Penta y SQM);

- casos de cobertura media y de interés regional: (casos Basura, Exalmar y Fipes).

Los casos estudiados son los siguientes:

- Caso Basura: se denuncian irregularidades en la contratación de servicios de retiro y depósito de basura en varios municipios de la Región Metropolitana. Interés local.

- Caso Caval: denuncias contra una sociedad formada por la nuera de la Presidenta de la República, Natalia Compagnon y su hijo, Sebastián Dávalos, que se ha visto involucrada en compra irregular de terrenos en la comuna de Machalí. Interés nacional.

- Caso Corpesca: denuncia de financiación irregular de campañas políticas del grupo Corpesca, vinculado al grupo Angelini, en el que aparecen involucrados el exministro Pablo Longueira y el senador Jaime Orpis.

- Caso Exalmar: inversiones de Bancard en la pesquera peruana Exalmar, en el contexto del litigio que mantenían Chile y Perú en el Tribunal Internacional de La Haya para determinar sus límites fronterizos en alta mar.

- Caso Fipes: financiación que habría recibido el diputado Iván Fuentes de parte de la Federación de Industrias Pesqueras del Sur Austral (Fipes).

- Caso Penta: grupo económico formado por los empresarios Carlos Delano y Carlos Eugenio Lavin, relacionado con financiación irregular de campañas políticas.

- Caso SQM: empresa minera, dirigida por el yerno de Augusto Pinochet, Julio Ponce Lerou, que estaría involucrada en la financiación irregular de campañas políticas mediante la emisión de facturas por servicios profesionales no prestados.

Hemos seleccionado una muestra de periódicos nacionales y regionales que constituye el corpus de análisis. Dentro de los medios regionales, se privilegió a aquellos periódicos con sede las ciudades donde sucedieron estos hechos de corrupción. Entre los medios de circulación nacional, los pertenecientes a la empresa periodística El Mercurio SAP, propietaria de

- El mercurio

- La segunda

- Las últimas noticias

Medios también pertenecientes a la empresa EI Mercurio SAP, pero de circulación regional:

- La estrella de Arica

- El mercurio de Valparaíso

- El llanquihue.

Por otro lado hemos incorporado tres periódicos pertenecientes al Consorcio Periodístico de Chile (Copesa SA),

- dos de circulación nacional: La tercera y La cuarta;

- uno de circulación regional: El diario de Concepción.

Finalmente hemos incorporado dos periódicos regionales independientes:

- El rancagüino, perteneciente a la Sociedad Informativa Regional;

- El diario de Aysén, de la Empresa Periodística de Aysén.

En total se trabajó con un universo de 10.011 items o unidades de análisis. Hemos accedido a este material desde el sistema Nex News. Para seleccionar las noticias que contienen las palabras clave y realizar la descarga, se consideró sólo el título, epígrafe y la bajada de cada noticia. Una vez que se descargó la información, se integró al análisis también el cuerpo de la noticia.

Con todo este material, hemos considerado al menos tres tipos de variables para el análisis:

- Noticias completas (título, epígrafe, bajada y cuerpo) descargadas para este estudio.

- Siete escándalos políticos identificados en el periodo 2014-2016.

- Once medios de prensa distribuidos en las diferentes regiones del país.

Para responder a P1 se hizo un ejercicio que permitió identificar los encuadres más presentes en la cobertura noticiosa cubierta por la muestra. Para P2 se realizó un análisis de la presencia de cada uno de estos encuadres para los escándalos estudiados. Para P3 se hizo un análisis por cada tipo de medio, intentando determinar si existía una conexión entre la cercanía y un mayor interés de los medios de prensa por informar sobre esos casos.

\section{Resultados}

En la tabla 1 se muestra la distribución de las noticias recolectadas según los periódicos y el escándalo político al que hacen referencia. Esta información está organizada según los grupos económicos a los que pertenece cada diario. Los porcentajes de noticias que publican los periódicos de un mismo grupo mediático para cada escándalo respecto al total de las noticias discrepa considerablemente. Así mismo, existen porcentajes similares para un mismo escándalo entre medios de grupos económicos diferentes. Por ejemplo, la proporción de noticias de El mercurio y La tercera en los 
escándalos estudiados es muy similar. La misma relación se observa entre Las últimas noticias y La cuarta, con leves variaciones. Esto nos hace pensar que, si bien la variable propiedad del grupo económico explica en parte el interés del periódico por cubrir ciertos escándalos políticos, lo cierto es que parece ser que la orientación nacional/regional es una variable más relevante para explicar su comportamiento frente a estos casos.

Tabla 1. Porcentaje de noticias publicadas por periódico para cada caso de estudio

\begin{tabular}{|c|c|c|c|c|c|c|c|c|c|c|c|}
\hline & \multicolumn{6}{|c|}{ EI Mercurio SAP } & \multicolumn{3}{|c|}{ Copesa SA } & \multicolumn{2}{|c|}{ Independientes } \\
\hline & 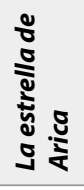 & 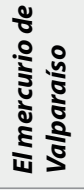 & 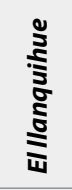 & 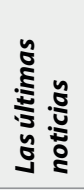 & 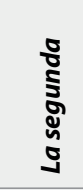 & 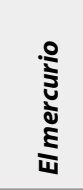 & 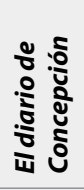 & 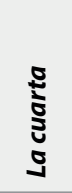 & 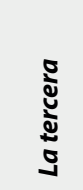 & 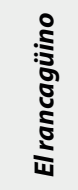 & 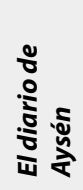 \\
\hline Basura & 0,8 & 2,8 & 1,7 & 0,7 & 2,3 & 1,6 & 1,0 & 1,8 & 1,7 & 2,1 & 2,3 \\
\hline Caval & 31,0 & 30,7 & 28,4 & 36,8 & 23,6 & 27,1 & 21,3 & 46,0 & 23,7 & 81,9 & 29,7 \\
\hline Corpesca & 17,5 & 7,4 & 7,5 & 7,0 & 7,7 & 7,5 & 8,6 & 13,3 & 7,4 & 2,1 & 15,1 \\
\hline Exalmar & 1,6 & 1,1 & 0,9 & 0,6 & 1,1 & 0,5 & 0,5 & 0,0 & 0,7 & 0,0 & 0,5 \\
\hline Fipes & 0,0 & 0,5 & 0,6 & 0,7 & 0,6 & 0,5 & 0,5 & 0,0 & 0,7 & 0,0 & 5,9 \\
\hline Penta & 34,9 & 29,6 & 36,4 & 23,7 & 29,5 & 30,1 & 36,5 & 16,8 & 28,2 & 11,7 & 31,1 \\
\hline$S Q M$ & 14,3 & 27,9 & 24,5 & 30,5 & 35,1 & 32,6 & 31,5 & 22,1 & 37,5 & 2,1 & 15,5 \\
\hline N. items & 126 & 638 & 535 & 544 & 1.579 & 2.951 & 197 & 113 & 3.015 & 94 & 219 \\
\hline
\end{tabular}

A partir de este corpus se realizó el análisis textual de cada uno de sus items. Se utilizó el programa Wordstat para identificar la co-ocurrencia de términos, esto es, cuándo dos palabras aparecen mencionadas en un mismo párrafo. En nuestro caso el software contabilizó en total 19 pares de términos. Estos pares de términos son diferentes a cero, según el índice de similaridad: coeficiente de Jaccard. El resultado de este proceso, es decir, los 20 grupos de pares de palabras y su presencia en los casos (\%), se presentan en la tabla 2. En la columna "Nombre", se muestra por defecto el conjunto de palabras seleccionadas, según las principales "Palabras clave" que aparecen en la respectiva columna. Por su parte, en la columna "\% casos", aparece la proporción en que ese grupo de palabras se encuentra presente en el total de las unidades de análisis.

Tabla 2. Pares de términos en co-ocurrencia

\begin{tabular}{|c|c|c|}
\hline Nombre & Palabras clave & $\%$ casos \\
\hline Seremi de vivienda; Valdés & Vivienda; Seremi; Valdés; Regulador; Modificación & 10,17 \\
\hline Superintendencia & Superintendencia; Valores; Bancos; SBIF; Financieras; Corpbanca & 10,21 \\
\hline Secreto bancario; Cuentas corrientes & Bancario; Secreto; Cuentas; Alzar; Corrientes; Bancarias & 11,63 \\
\hline Base imponible & Imponible; Base; Disminuir; Rebajar; Impuesto; Cónyuges; Utilización & 11,93 \\
\hline Negociación incompatible; Tráfico & Incompatible; Negociación; Tráfico; Influencias; Violación; Secreto; Enriquecimiento & 13,31 \\
\hline Cerda, cuñada; Subsecretario & Cuñada; Cerda; Subsecretario; Minería; Boleta & 14,16 \\
\hline Presunción de inocencia & Inocencia; Presunción; Derecho & 14,36 \\
\hline Arresto domiciliario; Nocturno & Arresto; Domiciliario; Nocturno; Arraigo; Cautelar; Cautelares; Quincenal; Medida & 16,31 \\
\hline Malversación; Caudales & Malversación; Caudales; Fisco; Fraude; Lavado; Ilícita; Activos; Asociación & 16,86 \\
\hline Maliciosamente incompletas; Inciso & $\begin{array}{l}\text { Incompletas; Inciso; Maliciosamente; Código; Tributario; Impuesto; Empleo; Sancio- } \\
\text { nado; Ocultar; Verdadero }\end{array}$ & 17,79 \\
\hline Juzgado de garantía & Garantía; Juzgado; Sobreseimiento; Suspensión; Definitivo & 20,67 \\
\hline Pena de presidio; Juicio oral & Pena; Oral; Presidio; Condicional; Juicio; Suspensión; Atenuantes; Procedimiento & 21,45 \\
\hline Bienes raíces & Raíces; Conservador; Bienes; Inscripción & 3,52 \\
\hline Ventajas indebidas & Indebidas; Ventajas; Devoluciones; Prevaricación & 3,92 \\
\hline Corfo & $\begin{array}{l}\text { Corfo; Pertenencias; Arbitral; Arrendamiento; Conciliación; Árbitro; Mineras; Explo- } \\
\text { tación; Estatal }\end{array}$ & 5,11 \\
\hline $\begin{array}{l}\text { Convenio preventivo; } \\
\text { Helga }\end{array}$ & Convenio; Preventivo; Helga; Acreedores; Insolvencia; Quiebras & 5,21 \\
\hline Apropiación indebida & Indebida; Apropiación; Facilitación & 6,59 \\
\hline Encuesta CEP; Adimark & Encuesta; CEP; Adimark; Aprobación; Evaluación; Positiva & 8,80 \\
\hline $\begin{array}{l}\text { Gestor inmobiliario; Obras de la munici- } \\
\text { palidad }\end{array}$ & Inmobiliario; Gestor; Municipalidad; Obras; Arquitecta & 9,29 \\
\hline Brigada & Brigada; Bridec; Económicos; PDI & 9,87 \\
\hline
\end{tabular}

Fuente: elaborado a partir de datos procesados con Wordstat 
Para lograr la caracterización adecuada de cada uno de los encuadres obtenidos es necesario agrupar aún más los pares de términos en co-ocurrencia. A partir de los 20 grupos de palabras, hemos inferido dos grandes grupos de términos en co-ocurrencia. Estos dos grupos representan 10 co-ocurrencias (en las primeras filas de la tabla 2) y 10 co-ocurrencias respectivamente (en las últimas filas de la tabla 2), dando cuenta del total del material analizado. Una vez que hemos separado estos dos grupos, haremos un análisis ya no de las co-ocurrencias, sino del resto del texto, gracias a la función "palabra clave en contexto", de Wordstat.

- Por un lado, identificamos un encuadre que estaría presente en el 51,51\% de los items analizados. Este grupo de términos hacen referencia en detalle al proceso judicial relacionado con cada escándalo. Este encuadre es utilizado para describir, principalmente, el período de investigación de los casos por parte del Ministerio Público, las garantías del debido proceso, así como las medidas que los respectivos juzgados van tomando hacia los protagonistas en las noticias. Por lo tanto, a este encuadre lo hemos denominado "judicialización de los casos".

- Por otro lado, encontramos un encuadre presente en el 52,40\% de los items analizados. Este grupo de términos hace referencia a la "atribución de responsabilidades" de cada caso. Si bien predominan categorías jurídicas, se refieren más a valoraciones negativas (malversación, malicioso, fraude, ilícito, etc.). El criterio de responsabilidad empleado aquí supera entonces el espacio de lo puramente jurídico. En lo fundamental dan cuenta de conflictos políticos, económicos, financieros y tributarios que parecen mezclarse. Aparecen también los actores y protagonistas de cada caso. Es decir, parece ser que este gran encuadre engloba varios encuadres: por un lado, las responsabilidades, las cuestiones de interés humano, la personalización y el botín conquistado. Por lo tanto, a este encuadre lo hemos denominado "atribución de responsabilidades", siguiendo en ello la definición genérica propuesta por Semetko y Valkenburg (2002). El detalle de las palabras clave que aparecen con mayor frecuencia en los items analizados se encuentran expresados en la tabla 3.

Tabla 3. Conceptos clave contenidos en cada encuadre

\begin{tabular}{|l|l|l|}
\hline \multicolumn{1}{|c|}{ Encuadre } & \multicolumn{1}{c|}{ Palabras clave } \\
\hline Judicialización de los casos & $\begin{array}{l}\text { Inocencia; Presunción; Derecho penal; Oral; Presidio; Condicional; Juicio; Suspensión; Ate- } \\
\text { nuantes; Procedimiento; Garantía; Juzgado; Sobreseimiento; Suspensión; Definitivo Arresto; } \\
\text { Domiciliario; Nocturno; Arraigo; Cautelar; Cautelares; Quincenal; Medida }\end{array}$ \\
\hline Atribución de responsabilidades & $\begin{array}{l}\text { Cuñada; Cerda; Subsecretario; Minería; Boleta; Indebidas; Ventajas; Devoluciones; Prevari- } \\
\text { cación; Malversación; Caudales; Fisco; Fraude; Lavado; Ilícita; Activos; Asociación; Incom- } \\
\text { pletas; Inciso; Maliciosamente; Código; Tributario; Impuesto; Empleo; Sancionado; Ocultar; } \\
\text { Verdadero; Imponible; Base; Disminuir; Rebajar; Impuesto; Cónyuges; Utilización; Indebida; } \\
\text { Apropiación; Facilitación; Bancario; Secreto; Cuentas; Alzar; Corrientes; Bancarias; Incompati- } \\
\text { ble; Negociación; Tráfico; Influencias; Violación; Secreto; Enriquecimiento }\end{array}$ \\
\hline
\end{tabular}

Fuente: elaborado a partir de datos procesados con Wordstat

\subsection{Presencia de encuadres en los escándalos políticos}

Hasta ahora hemos detectado dos encuadres utilizados por los medios de comunicación al hacer el tratamiento de los casos de escándalos políticos. La tabla 4 muestra la presencia de ambos encuadres para cada caso estudiado.

"Atribución de responsabilidades" es el encuadre que más presencia tiene en general en los casos estudiados, con un $61,2 \%$ del total. En cambio, "judicialización de los casos" es menor y alcanza un $38,79 \%$ de presencia en los casos. Las distribuciones son similares en los casos Fipes y Exalmar. La presencia del encuadre "Atribución de responsabilidades" es más importante en el caso Penta (23,33 contra 14,73\%), en Corpesca $(06,01$ contra $03,58 \%)$ o en el caso Basura $(01,87$ contra $01,04 \%)$. Una presencia similar se observa en los casos SQM (16,98 contra $10,79 \%)$ y Caval $(12,61$ contra 08,34\%).

Estas diferencias también se pueden observar en el gráfico 1. En azul se encuentra el área comprendida por el encuadre "atribución de responsabilidades" y en marrón el área comprendida por el encuadre "judicialización de los casos". No obstante, desde el punto de vista de la presencia de los encuadres en cada uno de los casos, resulta más semejante la cobertura que se dio a los casos SQM y Penta, mientras la cobertura del caso Caval se parece más a la cobertura dada a Corpesca.

También cabe señalar que el resto de los casos (Fipes, Exalmar, Basura) casi no aparecen representados en el gráfico, debido a que su presencia en los items es muy baja en comparación con otros.

Podemos entonces identificar al menos dos comportamientos según el tipo de casos estudiados:

Tabla 4. Distribución porcentual de los encuadres contenidos en la variable escándalos políticos

\begin{tabular}{|l|c|c|c|}
\cline { 2 - 4 } \multicolumn{1}{c|}{} & $\begin{array}{c}\text { Atribución de } \\
\text { responsabilidades }\end{array}$ & $\begin{array}{c}\text { Judicialización de } \\
\text { los casos }\end{array}$ & Total \\
\hline Basura & 1,87 & 1,04 & 2,91 \\
\hline Caval & 12,61 & 8,34 & 20,95 \\
\hline Corpesca & 6,01 & 3,58 & 9,59 \\
\hline Exalmar & 0,20 & 0,16 & 0,36 \\
\hline Fipes & 0,20 & 0,15 & 0,35 \\
\hline Penta & 23,33 & 14,73 & 38,06 \\
\hline SQM & 16,98 & 10,79 & 27,77 \\
\hline Total & 61,20 & 38,79 & 100 \\
\hline
\end{tabular}

Fuente: elaborado a partir de datos procesados con Wordstat 
- en el primer grupo (que incluye Caval, SQM, Corpesca, y Penta) el encuadre más presente es el de "atribución de responsabilidades". Esto se explica en parte por la fuerte presencia de actores políticos de primer orden (ministros, senadores, hijo de la presidenta). No obstante, existen diferencias significativas en la distribución total de los encuadres para cada caso. Es el caso de Caval, donde encontramos una presencia más alta del encuadre "judicialización de los casos" que en Penta y SQM.

- en el siguiente grupo de casos (Basura, Exalmar y Fipes) el encuadre "atribución de responsabilidades" se distribuye de manera más equilibrada en los items. Esto se explica en parte porque comprometen a personajes de segundo orden (alcaldes de Maipú, Cerro Navia y Ñuñoa, y diputados), con la sola excepción de Exalmar. La presencia del encuadre "judicialización de los casos" parece estar tan presente como "atribución de responsabilidades" en estos tres casos estudiados.

\section{Presencia de encuadres en los medios de prensa}

La presencia de los encuadres en los medios analizados se distribuye de manera uniforme en los medios de comunicación. La excepción la marcan los periódicos El mercurio, La cuarta, La segunda y La tercera, donde el encuadre "atribución de responsabilidades" predomina. La tabla 5 muestra la distribución de frecuencias por encuadre en cada medio analizado.

Para explicar la presencia de los encuadres en los medios de comunicación los separamos en dos grupos:

- un primer grupo compuesto por los medios con sede en Santiago: El mercurio, La tercera, Las últimas noticias (Lun), La segunda y La cuarta;

- un segundo grupo compuesto por la prensa regional, donde se incluye El diario de Aysén, El diario de Concepción, El Ilanquihue, El mercurio de Valparaíso, El rancagüino, y La estrella de Arica.

En azul se encuentra el área comprendida por el encuadre "atribución de responsabilidades" y en marrón el área comprendida por el encuadre "judicialización de los casos".

En el primer grupo de diarios, el encuadre que predomina -excepto en el caso de Las últimas noticias donde es más parejo el resultado- es "atribución de responsabilidades". Nuevamente, por las características del encuadre, es el más usado por los medios de circulación nacional.

Otro de los aspectos interesantes sobre los resultados es la similitud de los porcentajes en este primer grupo de medios, ya que en cuatro de los cinco diarios la proporción es dos tercios versus un tercio. En el siguiente apartado analizaremos el porqué de esta situación.

En el segundo grupo de medios de comunicación, compuesto por diarios regionales, la presencia de ambos encuadres noticiosos es más equitativa, aunque de igual forma predomina el encuadre "judicialización de los casos".

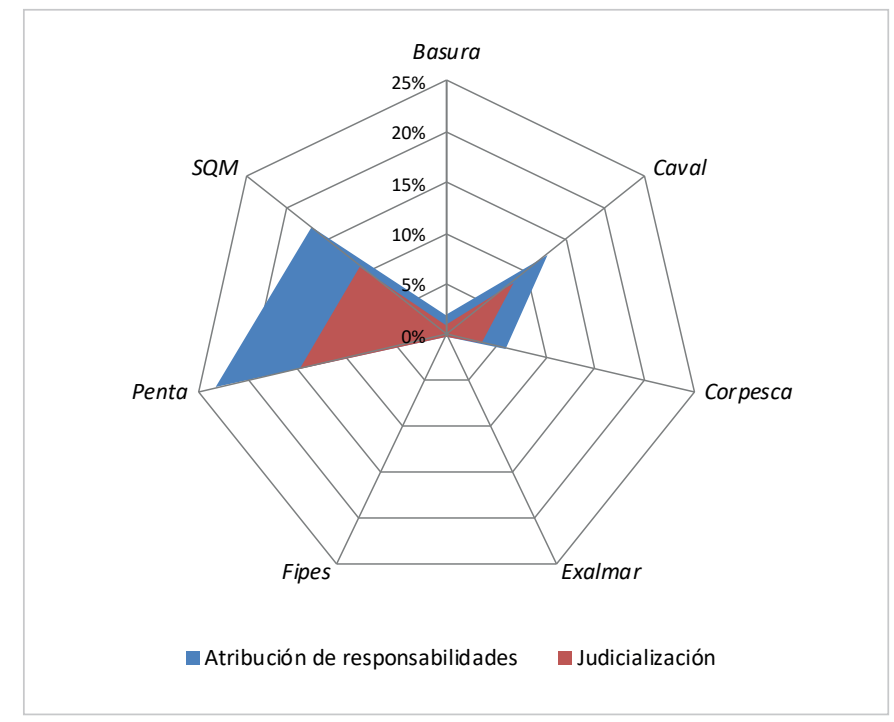

Gráfico 1. Presencia de encuadres noticiosos para cada escándalo político (\%). Fuente: elaborado a partir de datos procesados con Wordstat

Tabla 5. Distribución porcentual de los encuadres contenidos en las variables medios de comunicación

\begin{tabular}{|l|c|c|}
\cline { 2 - 3 } \multicolumn{1}{c|}{} & $\begin{array}{c}\text { Atribución de } \\
\text { responsabilidades }\end{array}$ & $\begin{array}{c}\text { Judicialización de } \\
\text { los casos }\end{array}$ \\
\hline La estrella de Arica & 50,19 & 49,81 \\
\hline El mercurio de Valparaíso & 55,65 & 44,35 \\
\hline El Ilanquihue & 55,40 & 44,60 \\
\hline Las últimas noticias & 50,67 & 49,33 \\
\hline La segunda & 62,13 & 37,87 \\
\hline El mercurio & 64,49 & 35,51 \\
\hline El diario de Concepción & 48,03 & 51,97 \\
\hline La cuarta & 61,96 & 38,04 \\
\hline La tercera & 62,89 & 37,11 \\
\hline El rancagüino & 52,58 & 47,42 \\
\hline El diario de Aysén & 52,33 & 47,67 \\
\hline
\end{tabular}

Fuente: elaborado a partir de datos procesados con Wordstat

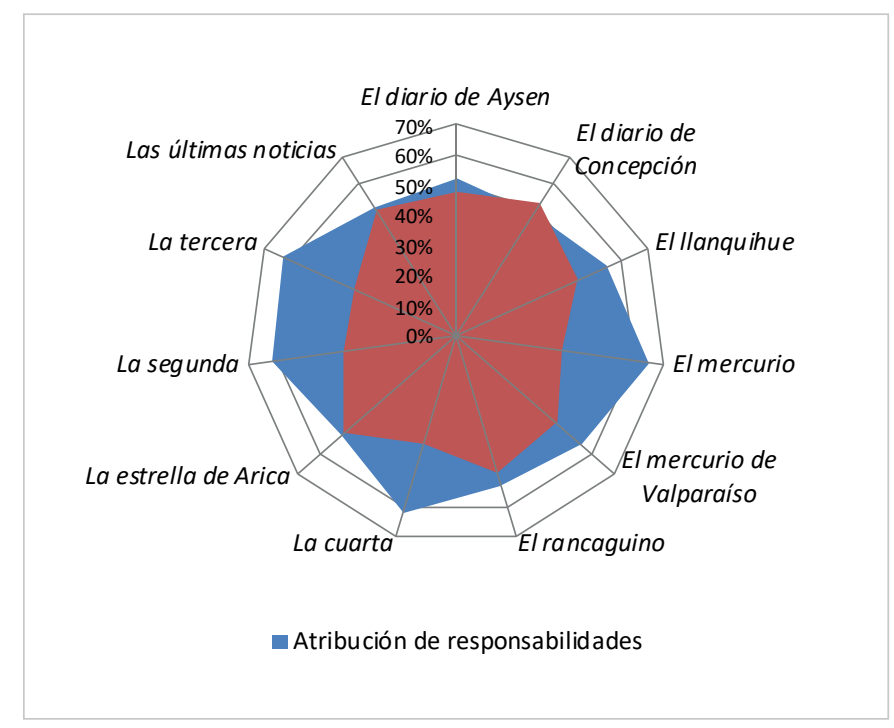

Gráfico 2. Presencia de encuadres noticiosos en cada periódico (\%). Fuente: elaborado a partir de datos procesados con Wordstat. 


\section{Conclusiones y discusión}

La sucesión de escándalos políticos en Chile durante los últimos años ha sido cubierta extensamente por los medios de comunicación. Las informaciones referidas a estos siete casos de estudio dan cuenta de ello. No obstante, la forma en que cada medio de comunicación presenta estas informaciones, si bien sigue pautas generales, da cuenta de diferentes formas de encuadrar las noticias.
La forma en que cada medio de comunicación presenta las informaciones de escándalos políticos, si bien sigue pautas generales, da cuenta de diferentes formas de encuadrar las noticias

Respecto a la pregunta de investigación 1, es posible señalar que los resultados del análisis dan cuenta de la presencia de dos grandes encuadres de noticias:

- encuadre "atribución de responsabilidades": pone el acento en establecer quiénes y de qué manera participan en los hechos. El encuadre parece integrar los elementos que están presentes en el encuadre genérico "conflicto", "personificación" y en el encuadre especifico "botín conquistado". El mapa de palabras hace referencia principalmente a las acusaciones hechas a los personajes y a las formas en que supuestamente se cometió el eventual ilícito.

- encuadre "judicialización de los casos": hace referencia a las diferentes aristas y etapas del proceso judicial. Se trata de un registro neutro, muy ajustado al lenguaje judicial, sobre el cual es difícil percibir juicios de valor y/o conflictos políticos o de índole moral. Este encuadre parece hacer mención a un proceso de judicialización de los problemas políticos que, al menos en Chile, parece haber tenido un carácter fallido (Couso, 2005), pero que hoy resurge con mayor énfasis debido a la cobertura intensiva de escándalos que realizan los medios de comunicación en la actualidad.

Respecto a la pregunta de investigación 2, podemos concluir que el encuadre que predomina es "atribución de responsabilidades". Con él lo que se busca es acentuar la búsqueda de los responsables. No obstante, la presencia del encuadre "Judicialización de los casos" es muy importante y cercana siempre al 50\%. El empleo del encuadre "atribución de responsabilidades" es más fuerte en los diarios de circulación nacional (El mercurio, La tercera y La cuarta) y también en diarios donde la cobertura política es más intensa ( La segunda). En el caso de los medios regionales, independiente del consorcio periodístico al cual pertenecen, la presencia de ambos encuadres es mucho más equilibrada.

Debido a la naturaleza de los personajes involucrados y a la cantidad de casos, la mayor o menor presencia de estos encuadres puede tener diversas explicaciones. La principal es la relevancia relativa de los protagonistas de las noticias. Debido a la gran cantidad de personajes y la importancia de sus cargos, se hace necesario recabar y dar a conocer cualquier antecedente -por parte de los medios- relevante respecto al hecho noticioso.
Hay dos grandes encuadres de noticias: "atribución de responsabilidades", que pone el acento en establecer quiénes participan en los hechos, y "judicialización de los casos", que hace referencia al proceso judicial

Respecto a la pregunta de investigación 3, constatamos que la cobertura que dan los medios de comunicación a los escándalos políticos, si bien sigue ciertas tendencias generales, no es en ningún caso totalmente homogénea. Mientras algunos periódicos publican noticias sobre escándalos diversos, otros ni siquiera los mencionan. Respecto al empleo de encuadres, se observa que su uso diferenciado expresa los énfasis que los diferentes medios de comunicación buscan imponer a los hechos. En términos generales, también podemos decir que se trata de una cobertura bastante centralista, que privilegia la publicación de artículos sobre escándalos políticos producidos en la capital. Lo regional no parece ser un factor relevante, salvo en el caso de El rancagüino. La diferencia más importante quizá se observe entre los medios con cobertura nacional y regional:

- en los medios con cobertura nacional, las rutinas o redacciones tienen una clara tendencia a emplear mucho más el frame "atribución de responsabilidades";

- los medios con cobertura regional no tienen una tendencia establecida como los anteriores, sino más bien se nutren de forma similar y equilibrada de ambos encuadres.

En conclusión, podemos señalar que la cobertura que la prensa chilena hizo de los escándalos políticos recientes fue bastante cauta, en parte por el carácter relativamente inédito de estos casos. La forma en que la prensa matiza o acentúa la gravedad de estos hechos parece estar relacionada con el menor o mayor uso del encuadre "judicialización de los casos". La distribución de noticias encontradas da cuenta además de una cierta tendencia de los medios a dar mayor cobertura a hechos políticos con protagonistas relevantes antes que a otro tipo de escándalos. Finalmente, la cuestión de la centralización de la agenda mediática, incluso entre los medios regionales, representa una tendencia muy fuerte y que conspira contra una información más cercana a las realidades de los públicos de las regiones del país.

Mientras algunos periódicos publican noticias sobre escándalos políticos diversos, otros ni siquiera los mencionan 


\section{Referencias}

Allern, Sigurd; Pollack, Ester (eds.) (2012). Scandalous! The mediated construction of political scandals in four Nordic countries. Gothenburg, SE: Nordicom. ISBN 9789186523275

Allern, Sigurd; Von-Sikorski, Cristian (2018). "Political scandals as a democratic challenge: From important revelations to provocations, trivialities, and neglect - Introduction". International journal of communication, v. 12.

https://ijoc.org/index.php/ijoc/article/view/7094

Arriagada, Arturo; Correa, Teresa; Scherman, Andrés; Abarzúa, Josefina (2015). "Santiago no es Chile: brechas, prácticas y percepciones de la representación medial en las audiencias chilenas". Cuadernos.info, n. 37, pp. 63-75.

https://doi.org/10.7764/cdi.37.769

CEP Chile (2014). Estudio nacional de opinión pública, noviembre 2014.

https://www.cepchile.cl/estudio-nacional-de-opinion-publica-noviembre-2014/cep/2016-03-04/100954.html

CEP Chile (2015). Estudio nacional de opinión pública, abril 2015.

https://www.cepchile.cl/estudio-nacional-de-opinion-publica-abril-2015/cep/2016-03-04/101227.html

CEP Chile (2016). Estudio nacional de opinión pública, julio-agosto 2016.

https://www.cepchile.cl/estudio-nacional-de-opinion-publica-julio-agosto-2016/cep/2016-08-18/165239.html

Couso, Javier A. (2005). "The judicialization of Chilean politics: The rights revolution that never was". In: Sieder Rachel; Schjolden, Line; Angell, Alan (eds.). The judicialization of politics in Latin America. Studies of the Americas. New York: Palgrave Macmillan, pp. 105-129. ISBN: 9780230619692

https://doi.org/10.1007/978-1-137-10887-6_5

De-Vreese, Claes H. (2005). "News framing: Theory and typology". Information design journal, v. 13, n. 1, pp. 51-62. https://doi.org/10.1075/idjdd.13.1.06vre

Fenoll, Vicente; Rodríguez-Ballesteros, Paula (2017). “Análisis automatizado de encuadres mediáticos. Cobertura en prensa del debate 7D 2015: el debate decisivo". El profesional de la información, v. 26, n. 4, pp. 630-640.

https://doi.org/10.3145/epi.2017.jul.07

Fernández-Vázquez, Pablo; Barberá, Pablo; Rivero, Gonzalo (2016). "Rooting out corruption or rooting for corruption? The heterogeneous electoral consequences of scandals". Political science research and methods, v. 4, n. 2, pp. 379-397. https://doi.org/10.1017/psrm.2015.8

Gamboa, Ricardo; Segovia, Carolina (2016). “Chile 2015: Falla política, desconfianza y reforma”. Revista de ciencia política, v. 36, n. 1, pp. 123-144.

https://scielo.conicyt.cl/pdf/revcipol/v36n1/art06.pdf

Gronemeyer, María-Elena; Porath, William (2015). “Un studio de la homogeneidad entre las posturas editoriales y de las fuentes informativas en la prensa de referencia chilena". Cuadernos.info, n. 36, pp.139-153.

https://doi.org/10.7764/cdi.36.567

Hallin, Daniel C.; Mellado, Claudia (2017). "Serving consumers, citizens, or elites: Democratic roles of journalism in Chilean newspapers and television news". The international journal of press/politics, v. 23, n. 1, pp. 24-43.

https://doi.org/10.1177/1940161217736888

Igartua, Juan-José; Muñiz-Muriel, Carlos; Cheng, Lifen (2005). “La inmigración en la prensa española. Aportaciones empíricas y metodológicas desde la teoría del encuadre noticioso". Migraciones, n. 17, pp. 143-181.

https://revistas.comillas.edu/index.php/revistamigraciones/article/view/4220

Jacobsson, Kerstin; Löfmark, Erik (2008). "A sociology of scandal and moral transgression: The Swedish 'Nannygate' scandal". Acta sociologica, v. 51, n. 3, pp. 203-216.

https://doi.org/10.1177/0001699308094166

Markovitz, Andrei S.; Silverstein, Mark (1988). The politics of scandal: Power and progress in liberal democracies. New York, NY: Holmes \& Meie. ISBN: 9780841910980

Matthes, Jörg; Kohring, Matthias (2008). "The content analysis of media frames: Toward improving reliability and validity". Journal of communication, v. 58, n. 2, pp. 258-279.

https://doi.org/10.1111/j.1460-2466.2008.00384.x

Mellado-Ruiz, Claudia; Humanes, María-Luisa (2017). “Homogeneity and plurality of the media agenda in Chile. A crosslongitudinal study of the national print press between 1990 and 2015". Communication \& society, v. 30, n. 3, pp. 75-92. https://revistas.unav.edu/index.php/communication-and-society/article/view/35770 
Mellado-Ruiz, Claudia; López-Rabadán, Pablo; Elortegui, Claudio (2017). “¿(Para) ciudadanos o espectadores? Periodismo político chileno y sus modelos profesionales de aproximación a la audiencia”. Palabra clave, v. 20, n. 1, pp. 14-46. https://doi.org/10.5294/pacla.2017.20.1.2

Miller, M. Mark (1997). "Frame mapping and analysis of news coverage of contentious issues". Social science computer review, v. 15, n. 4, pp. 367-378.

https://doi.org/10.1177/089443939701500403

Mönckeberg, María-Olivia (2009). Los magnates de la prensa. Concentración de los medios de comunicación en Chile. Random House, Santiago de Chile. ISBN: 9789568410292

Navia, Patricio; Osorio, Rodrigo (2015). "El mercurio lies, and La tercera lies more. Political bias in newspaper headlines in Chile, 1994-2010". Bulletin of Latin American research, v. 34, n. 4, pp. 467-485.

https://doi.org/10.1111/blar.12364

Palau, Anna; Davesa, Ferran (2013). “El impacto de la cobertura mediática de la corrupción en la opinión pública española". Revista española de investigaciones sociológicas, n. 144, pp. 97-126.

https://doi.org/10.5477/cis/reis.144.97

Puglisi, Riccardo; Snyder, James M. (2011). "Newspaper coverage of political scandals". The journal of politics, v. 73, n. 3, pp. 931-950.

https://www.jstor.org/stable/10.1017/s0022381611000569

Romano, María-Belén (2016). “Escándalo político y prensa escrita en Argentina. Un estudio desde el análisis crítico del discurso". Discurso \& sociedad, n. 10, n. 1, pp. 52-77. http://www.dissoc.org/ediciones/v10n01/DS10\%281\%29Romano.pdf

Salinas-Muñoz, Claudio; Jara-Reyes, René-Eduardo; Stange-Marcus, Hans; Del-Valle-Rojas, Carlos (2015). "Los desafíos de la integración y el pluralismo: la prensa nacional y regional en Chile". Chasqui, n. 130, pp. 313-328.

https://bit.ly/2P2E1Hm

Semetko, Holli A.; Valkenburg, Patti M. (2002). "Framing European politics: A content analysis of press and television news". Journal of communication, v. 50, n. 2, pp. 93-109.

https://doi.org/10.1111/j.1460-2466.2000.tb02843.x

Stange-Marcus, Hans; Salinas-Muñoz, Claudio; Yáñez-Duamante, Constanza; Santa-Cruz-Achurra, Eduardo (2018). "Pluralismo informativo y burocratización del trabajo periodístico en dos contextos de prensa diaria". Estudios sobre el mensaje periodístico, v. 24, n. 2, pp. 1825-1843.

https://doi.org/10.5209/ESMP.62249

Stein-de-Sparvieri, Elena (2013). "La corrupción política y su expresión en el discurso periodístico". Subjetividad y procesos cognitivos, v. 17, n. 2, pp. 133-155.

https://dialnet.unirioja.es/servlet/articulo?codigo $=5126747$

Sunkel, Guillermo (2004). "Narrativas periodísticas y escándalos políticos: el caso Mop-Ciade". Comunicación y medios, n. 15 , pp. $85-100$

https://doi.org/10.5354/0719-1529.2011.12084

Sunkel, Guillermo; Geoffroy, Esteban (2001). Concentración económica de los medios de comunicación. LOM Ediciones: Santiago. ISBN: 9562824152

Thompson, John B. (2001). El escándalo político. Poder y visibilidad en la era de los medios de comunicación, Barcelona: Paidós. ISBN: 8449311608

Valenzuela, Sebastián; Arriagada, Arturo (2009). "Competencia por la uniformidad en noticieros y diarios chilenos, 2000-2005". Cuadernos de información, n. 24, pp. 41-52.

http://www.cuadernos.info/index.php/CDI/article/view/33/30

Von-Sikorski, Cristian (2018). "Political scandals as a democratic challenge. The aftermath of political scandals: A meta-analysis". International journal of communication, v. 12, n. 25

https://ijoc.org/index.php/ijoc/article/view/7100

Von-Sikorski, Cristian; Knoll, Johannes (2019). "Framing political scandals: Exploring the multimodal effects of isolation cues in scandal news coverage on candidate evaluations and voting intentions". International journal of communication, v. 13, n. 23. https://ijoc.org/index.php/ijoc/article/view/9616

Zamora, Rocío; Marín-Albaladejo, Juan-Antonio (2010). "La representación simbólica del escándalo político. Hacia una tipología de los marcos periodísticos (frames) utilizados en la narración del escándalo de corrupción política". Razón y palabra, v. 15, n. 73.

http://razonypalabra.org.mx/N/N73/Varia73/34ZamoraMarin_V73.pdf 